\title{
A broadband CPW-fed dual circularly polarized slot antenna with modified L-shaped monopoles
}

\author{
Linli Jiang ${ }^{1 a)}$, Chunlan Lu ${ }^{1}$, Wenquan Cao ${ }^{1}$, and Ligong Sun ${ }^{2}$ \\ ${ }^{1}$ College of Communications Engineering, Army Engineering University of PLA, \\ Nanjing 210007, China \\ ${ }^{2}$ Communication NCO Academy, Army Engineering University of PLA, \\ Chongqing 400000, China
}

a)15651779122@163.com

Abstract: In this letter, a novel broadband dual circularly polarized (CP) square slot antenna based on coplanar waveguide (CPW) feed is presented. Two orthogonal modified L-shaped monopoles fed by the CPW structure are designed to achieve dual circularly polarization. Through digging several rectangular notches while adding extra needle shaped tuning strip between two monopoles, wide impedance bandwidth (IMBW), wide axial ratio bandwidth (ARBW) and high port isolation are realized. Finally, in order to verify the analysis, a prototype is fabricated and measured. The measured available $-10 \mathrm{~dB}$ IMBW of each port is $76 \%$ (from $1.8 \mathrm{GHz}$ to $4.0 \mathrm{GHz}$ ), which covers the whole $3 \mathrm{~dB}$ ARBW of $67 \%$ (from $1.87 \mathrm{GHz}$ to $3.75 \mathrm{GHz}$ ). Besides, the port isolation is below $-25 \mathrm{~dB}$ and the cross polarization level is over $-15 \mathrm{~dB}$ across the entire operating band, which proves the proposed antenna a good candidate for communication systems.

Keywords: broadband, CPW, dual circularly polarization, slot antenna Classification: Microwave and millimeter-wave devices, circuits, and modules

\section{References}

[1] Q. Luo, et al:: "Wideband multilayer dual circularly polarised antenna for array application,” Electron. Lett. 51 (2015) 2087 (DOI: 10.1049/el.2015.3343).

[2] J. Lin, et al.: "AMC-based planar antenna with low-profile and broad CP beamwidth," IEICE Electron. Express 14 (2017) 20170473 (DOI: 10.1587/ elex.14.20170473).

[3] L. S. Yang, et al:: "CPW-fed slot antenna for UWB short-range impulse radar systems," IEICE Electron. Express 9 (2012) 1604 (DOI: 10.1587/elex.9.1604).

[4] J. S. Row: "The design of a squarer-ring slot antenna for circular polarization," IEEE Trans. Antennas Propag. 53 (2005) 1967 (DOI: 10.1109/TAP.2005. 848482).

[5] S.-W. Zhou, et al:: "A CPW-fed broadband circularly polarized regularhexagonal slot antenna with L-shape monopole," IEEE Antennas Wireless Propag. Lett. 10 (2011) 1182 (DOI: 10.1109/LAWP.2011.2172570). 
[6] R. Cao and S. C. Yu: "Wideband compact CPW-fed circularly polarized antenna for universal UHF RFID reader," IEEE Trans. Antennas Propag. 63 (2015) 4148 (DOI: 10.1109/TAP.2015.2443156).

[7] J.-Y. Sze, et al.: "Broadband CPW-fed circularly polarized square slot antenna with lightening-shaped feedline and inverted-L grounded strips," IEEE Trans. Antennas Propag. 58 (2010) 973 (DOI: 10.1109/TAP.2009.2039335).

[8] M. Yadav, et al:: "A compact offset CPW-fed dual polarized stubs loaded monopole antenna for quad-band operation," Proc. 8th International Conference on Computing, Communication and Networking Technologies (2017) 1 (DOI: 10.1109/ICCCNT.2017.8204128).

[9] C. S. Wu, et al:: "A CPW-fed slot antenna with dual band and dual circular polarization,” Proc. ISAP2016 (2016) 810.

[10] R. K. Saini and S. Dwari: "A broadband dual circularly polarized square slot antenna,” IEEE Trans. Antennas Propag. 64 (2016) 290 (DOI: 10.1109/TAP. 2015.2496118).

[11] G. S. Reddy, et al.: "Dual orthogonal CPW feed single element UWB antenna with polarization diversity," Proc. Eur. Radar Conf. (2015) 1411 (DOI: 10. 1109/EuRAD.2015.7346319).

\section{Introduction}

CP antenna, with its advantages of alleviating polarization mismatch losses and multipath effect, has been widely used in the modern wireless communication systems [1,2]. Performances such as low-profile, wide operating bandwidth and polarization diversity are taken into consideration in antennas design as well. Compared with traditional antennas, CPW-fed slot antennas exhibit more advantages due to the CPW structure and slot structure, which not only obtain broader operating bandwidth but also be easily integrated with active devices [3, 4]. Therefore, CPW-fed CP slot antennas play a more and more important role in wireless communication application.

In $[5,6,7]$, all proposed antennas are CPW-fed square slot antennas, and all of them introduce L-shaped strips to work as perturbation structures, each of them excites two orthogonal resonant modes so that $\mathrm{CP}$ wave can be radiated. Through adding inverted grounded strips, bandwidth can be improved in some degree but still not enough to meet the demands for high speed and giant capacity communication. Dual CP CPW-fed slot antennas, due to their properties of frequency reuse, communication capacity enhancement and polarization diversity, have been proposed and analyzed in many researches $[8,9,10]$. In [8], a CPW-fed dualpolarized stub loaded monopole antenna is proposed, it operates at four bands and excites both linearly polarization (LP) and CP. A CPW-fed slot antenna with dual band dual CP is invested in [9], with its novel inverted-annular shaped structure, it can obtain left handed circularly polarization (LHCP) at $2.1 \mathrm{GHz}$ and right handed circularly polarization (RHCP) at $2.9 \mathrm{GHz}$. In [10], a dual CP square slot antenna is proposed, which has a $60 \% 3 \mathrm{~dB}$ ARBW and the isolation is below $-15 \mathrm{~dB}$ within the bandwidth. As for port isolation enhancement, through adjusting the length of the slanted strips, the low mutual coupling is realized [11]. 
In this letter, a novel broadband CPW-fed dual CP slot antenna is proposed. It is made up of two orthogonal modified L-shaped monopoles based on the CPW structure, a needle tubing shaped tuning strip and several notches. All the components are located in one substrate. Results show that the proposed antenna can excite LHCP and RHCP simultaneously. Meanwhile, the proposed antenna has a relatively small size, low profile and simple feeding structure. The detailed design of proposed antenna is shown in Section II. The simulated and measured results are studied in Section III. Finally, conclusions are elaborated in Section IV.

\section{Antenna design and analyze}

\subsection{Configuration of antenna and design process}

Fig. 1 shows the configuration of the dual CP square slot antenna, which is fed by a $50-\Omega$ CPW feed with a signal strip line. The antenna consists of two orthogonal modified L-shaped monopoles, a strip with the shape of needle is connected to the left lower corner and is $+x$ directed with an angle of $45^{\circ}$, and several pairs of notches are dug from the ground. Dual CP are obtained by the L-shaped monopole, improvement of the bandwidth and port isolation are implemented by the slant needle shaped strip and notches. When one L-shaped monopole is working as the feed line, another monopole plays a role of stub. In this situation, one kind of CP wave is radiated. When it comes to another port, another type of CP wave can be realized as well. Since the symmetrical structure, only simulated results of port 1 are given in the designing and optimizing process. All the components are printed on an FR4 (relative permittivity 4.4, loss tangent 0.002 ) substrate material with the thickness of $1.6 \mathrm{~mm}$, detailed dimensions of the parameters are given in Fig. 1.
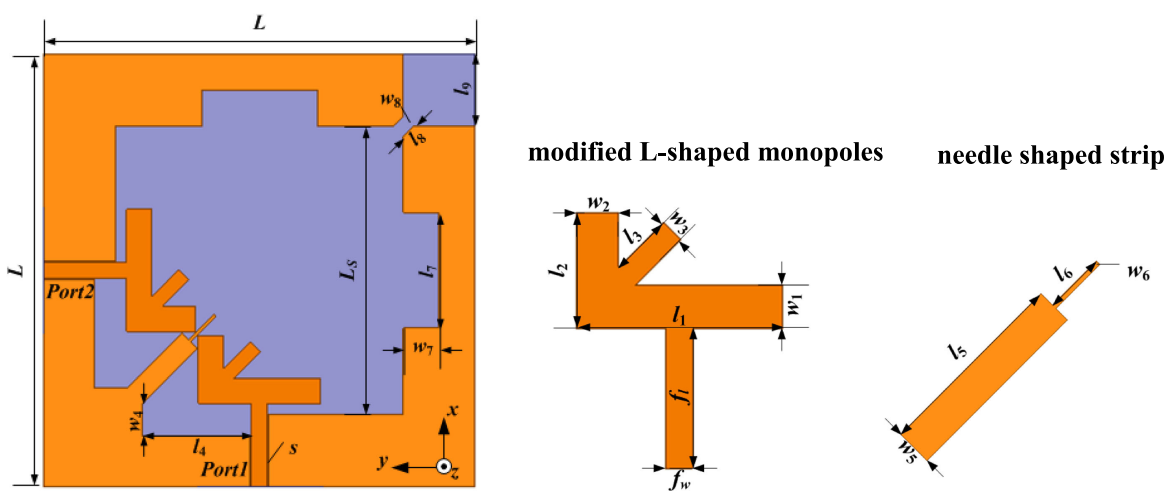

Fig. 1. Geometry of proposed antenna. $\left(L=60, L_{S}=40, f_{w}=2.2\right.$, $f_{l}=11.5, \quad s=0.2, l_{1}=17, w_{1}=3.5, l_{2}=9.5, \quad w_{2}=3.5$, $l_{3}=5.2, w_{3}=2, l_{4}=15, w_{4}=2, l_{5}=10.8, w_{5}=3, l_{6}=5$, $w_{6}=0.4, l_{7}=16, w_{7}=5, l_{8}=2, w_{8}=1$ and $l_{9}=10$ ) (unit: millimeter).

To elaborate the design procedure of the proposed antenna, four steps depicting the evolution structure are shown in Fig. 2. Simulated S-parameters, axial ratio results of the four antennas are revealed in Fig. 3. Ant. 1 is a square slot antenna with two modified L-shaped monopoles etched on the substrate along $+x$ axis and $-y$ axis respectively, each monopole is composed of a basic L-strip and a slant stub located at the right-angle. Two orthogonal currents are generated in the two arms of 


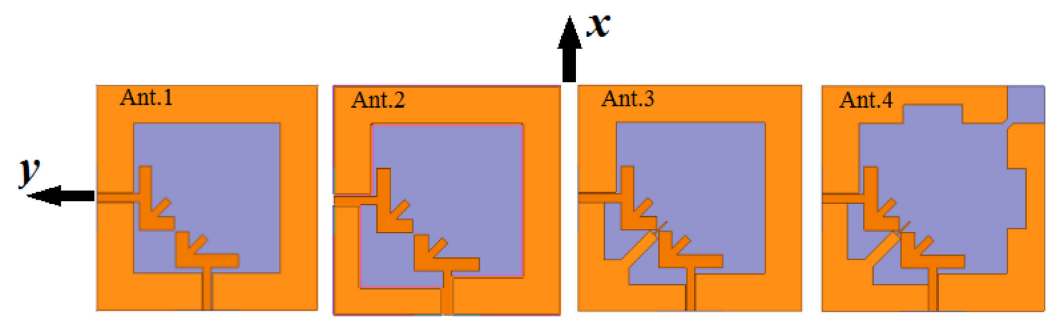

Fig. 2. The development of the antenna's design.

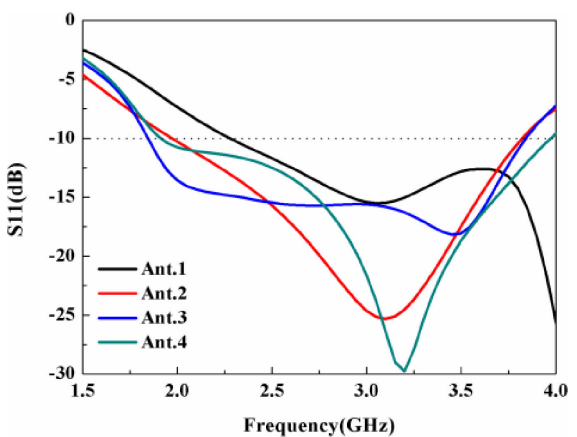

(a)

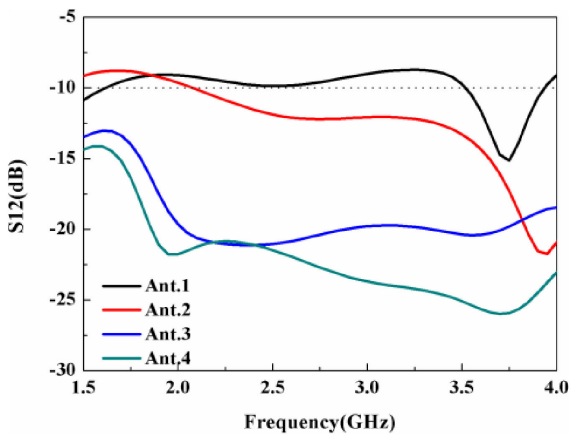

(b)

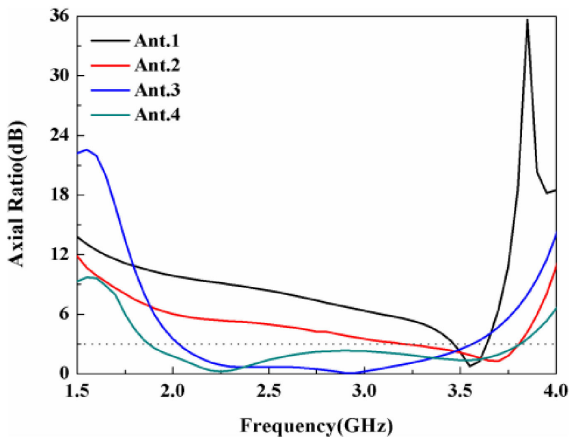

(c)

Fig. 3. Simulated results of the (a) $S_{11}$, (b) $S_{12}$ and (c) axial ratio of Ant. 1 to Ant. 4.

the monopoles to generate $\mathrm{CP}$ wave. From Fig. 3, Ant. 1 achieves a broad $-10 \mathrm{~dB}$ IMBW but the port isolation is only around $-10 \mathrm{~dB}$ and merely radiates $\mathrm{CP}$ wave at 3.55 GHz. It is seen in Ant. 2 that two notches are embedded from the left lower ground near the $\mathrm{CPW}$-fed line, in this method, the operating frequency moves to lower band, port isolation improves at higher frequency and a slightly wider $3 \mathrm{~dB}$ ARBW is realized. Reason for the frequency moving is that the mid-frequency 
depends on the length of the slot, which is set approximately half of the guided wavelength of the middle frequency, and it equals to be prolonged after notching thus the frequency moves to lower frequency in return. The ARBW improvement principal is that after notching current distribution in the short arm and the joint of two arms is more regular [5]. In order to achieve wider bandwidth, Ant. 3 is designed by adding a needle shaped strip at the left lower corner of the ground. As it can be drawn in Fig. 3, Ant. 3 exhibits much better port isolation and wider bandwidth than Ant. 2. This variation can be explained in two aspects. For the macroscopic side the slant strip can be treated as a perturbation and resonant at other frequency thus broadening the bandwidth. As for the microcosmic way, employment of the slant strip adjusts the currents in L-shaped monopole so that amplitudes of the two arms tend to be equal and excites better CP wave. Compared to Ant. 3, Ant. 4 notches the ground further, a pair of rectangles is embedded along the upper and right side of the slot and another pair of small notches is notched from the right upper corner. It can be observed in Fig. 3 that in this method the two ports divide further more from each other so that coupling between them becomes weaker, now the operating bandwidth doesn't change but better port isolation is obtained, the antenna's impedance matching and $3 \mathrm{~dB}$ ARBW get improved as well.
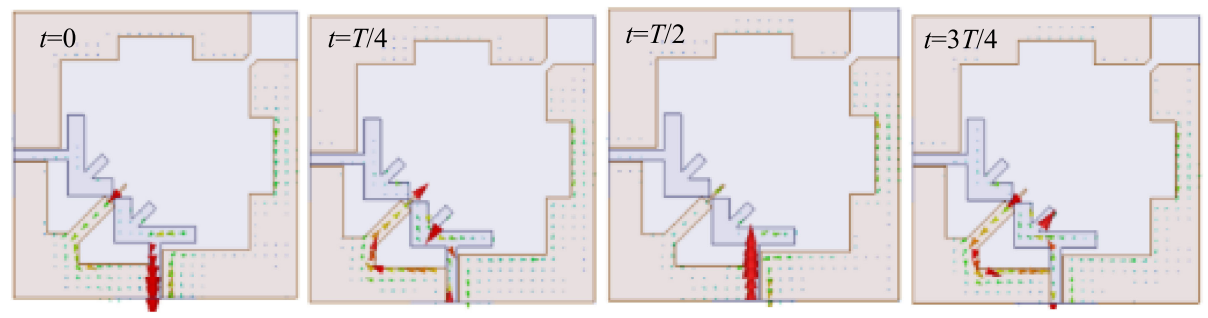

(a)
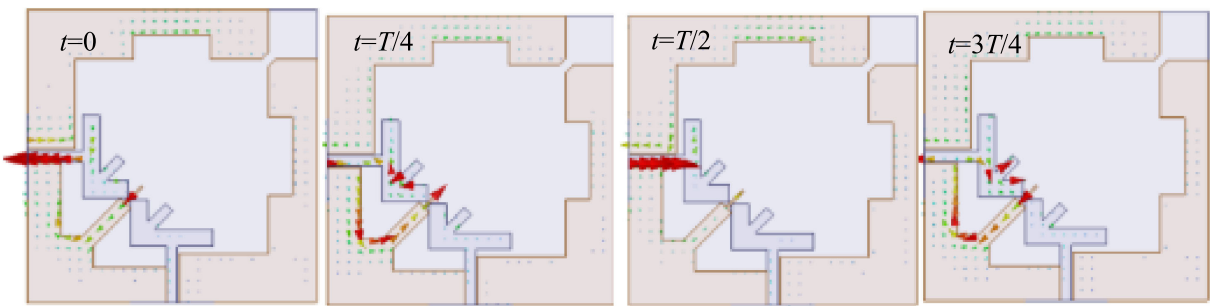

(b)

Fig. 4. Current distributions of the proposed antenna at $3 \mathrm{GHz}$ for (a) port 1 and (b) port 2 during a period.

Current distributions of the proposed antenna at $3 \mathrm{GHz}$ for both port 1 and port 2 with a period $T$ are shown in Fig. 4 . When port 1 is excited, it is obviously found that in Fig. 4(a) the surface current rotates anticlockwise as the time increases, as a consequence RHCP wave along $+z$ axial and LHCP wave along $-z$ axial are radiated. Fig. 4(b) elaborates that clockwise current rotation creates LHCP wave and RHCP wave along $+z$ and $-z$ axial respectively. What's more, it is worth mentioning that from current distributions at $t=T / 4$ and $t=3 T / 4$ for both ports, current is distributed along the slant stub, which in another side demonstrates 
the introduction of the needle shaped stub contributes to produce the $\mathrm{CP}$ wave and provide a novel thought for bandwidth improvement.

\section{2 parameters optimization}

For the purpose of achieving better performances for the proposed dual CP antenna, several parameters optimization have been carried out. By adjusting different parameters one by one to find their impacts on the antenna, the optimized antenna size can be selected. The influence of the L-strip's two arms on the antenna's performances have been studied in previous researches. However, in this letter, since the novel structure of modified L-shaped monopoles and needle shaped strip, more concerns are attached on the additional short arm and needle strip, which plays a role of fine tuning structure. Inspired by the current distributions in Fig. 4, two parameters are optimized and prove crucial to improve port isolation and bandwidth at last. One of them is $l_{3}$, which represents the length of the added stubs that connected with the L shaped monopoles, Fig. 5(a) and Fig. 5(b) imply that with the variation of $l_{3}$ from $5.2 \mathrm{~mm}$ to $9.2 \mathrm{~mm}, S_{12}$ meliorates in some degree, nevertheless the axial ratio moves higher in the middle frequency so that operating bandwidth becomes narrower. Another parameter to be optimized is $l_{5}, l_{5}$ measures the length of the needle tuning connected with the left lower corner, it is obviously unveils in Fig. 6(a) and Fig. 6(b) that when $l_{5}$ decreases from $10.8 \mathrm{~mm}$ to $6.8 \mathrm{~mm}$, both port isolation and $3 \mathrm{~dB}$ ARBW get deteriorated. Consider taking a balance between the port isolation and bandwidth, the finally optimized $l_{3}$ and $l_{5}$ are $5.2 \mathrm{~mm}$ and $10.8 \mathrm{~mm}$ respectively. Therefore, more flexible and convenient adjustment is allowed.

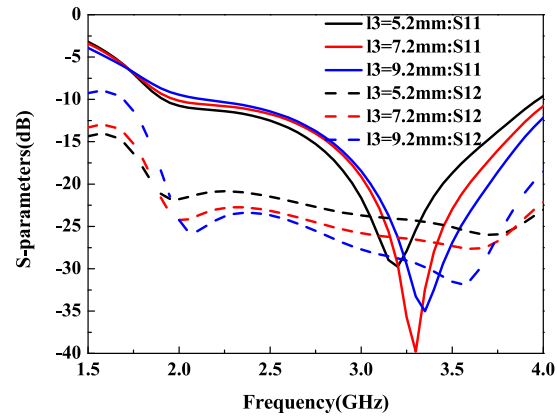

(a)

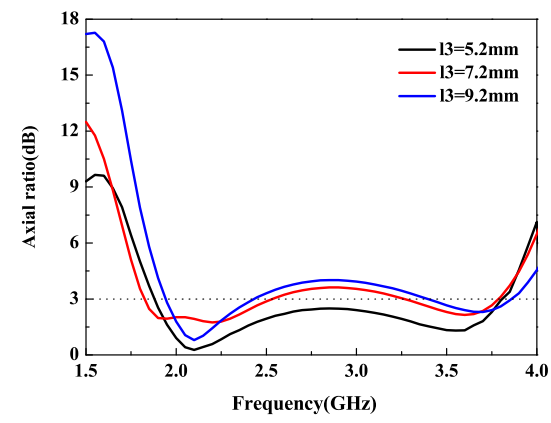

(b) 


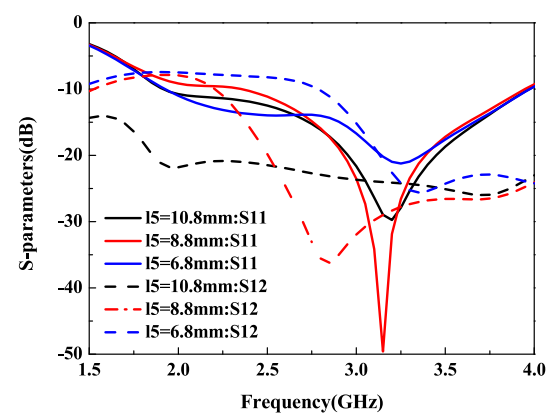

(a)

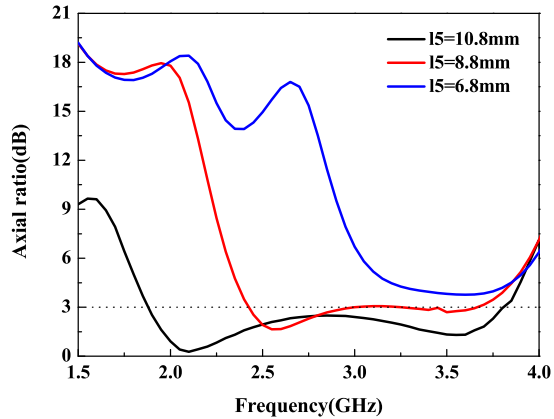

(b)

Fig. 6. Simulated results of (a) S-parameters and (b) axial ratio with the variation of $l_{5}$.

\section{Fabrication and measurement}

The proposed antenna is fabricated and tested to verify the analysis and the prototype is shown in Fig. 7. Fig. 8 displays the simulated and measured Sparameters results, the measured $-10 \mathrm{~dB}$ IMBW is $2.25 \mathrm{GHz}$ (from $1.75 \mathrm{GHz}$ to $4.0 \mathrm{GHz}$ ) for port 1 and $2.2 \mathrm{GHz}$ (from $1.8 \mathrm{GHz}$ to $4.0 \mathrm{GHz}$ ) for port 2 respectively, and the measured port to port isolation is below $-25 \mathrm{~dB}$ in the operating band. As it is seen in Fig. 9, when port 1 is excited and port 2 is matched, a broad 67\% (from $1.87 \mathrm{GHz}$ to $3.75 \mathrm{GHz}$ ) measured $3 \mathrm{~dB}$ ARBW is realized; when it comes to port 2, the measured ARBW overlaps with that of port 1, which can be easily explained for the antenna's symmetrical structure. Far field simulated and measured radiation patterns for both ports at $1.9 \mathrm{GHz}$ and $3.7 \mathrm{GHz}$ are given in Fig. 10. When port 1 is excited, the antenna is RHCP in $+z$ direction and radiates LHCP wave in $-z$ direction; $+z$ direction's LHCP and $-z$ direction's RHCP wave are radiated when exciting port 2 . The crossing polarization is below $-15 \mathrm{~dB}$ at all this situations. For both $1.9 \mathrm{GHz}$ and $3.7 \mathrm{GHz}$ frequencies the antenna manifests similar radiation patterns, therefore the antenna is proved to be a wide bandwidth dual $\mathrm{CP}$ antenna. Fig. 11 shows the simulated and measured gain against frequency, which is more than $1.4 \mathrm{~dB}$ in the operating band. All of the simulated and measured results above are in accordance with each other except Fig. 10(e) and Fig. 10(h), which have a relatively difference between the measured and simulated results. This small discrepancy can be explained in two aspects. One is the processing tolerance of the two ports, which leads to discrepancy in different planes when exciting different port. Another is the antenna's higher sensitivity in higher frequency towards the experimental environment included cable, SMA connectors and installation of 
equipment. Besides, performances comparison with several existing antennas in references are listed in Table I, it reveals that the proposed CPW-fed dual CP slot antenna does exhibit relatively compacter size, broader bandwidth and higher port isolation, thereby it is more favorable than other antennas in Table I.

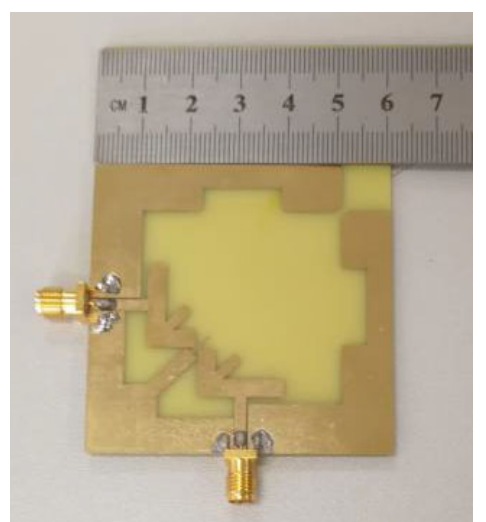

Fig. 7. Prototype of the proposed antenna.

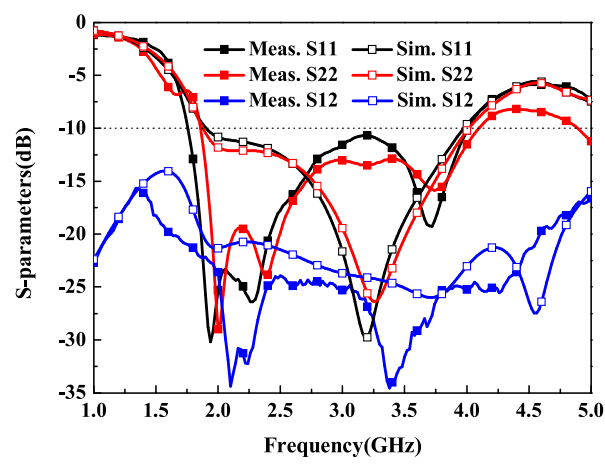

Fig. 8. Simulated and measured S-parameters results of the proposed antenna.

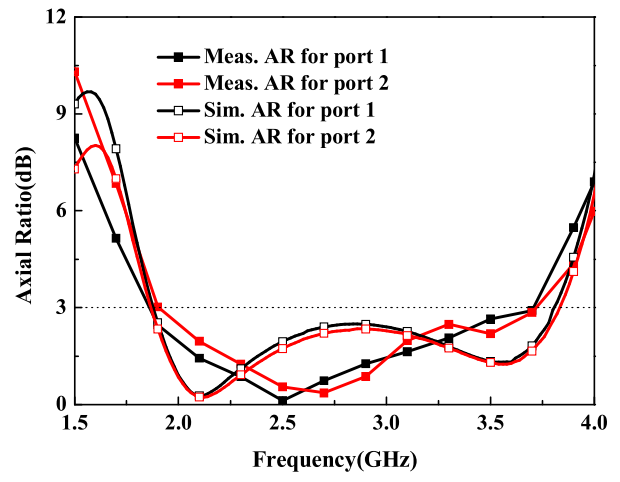

Fig. 9. Simulated and measured axial ratio results of the proposed antenna. 


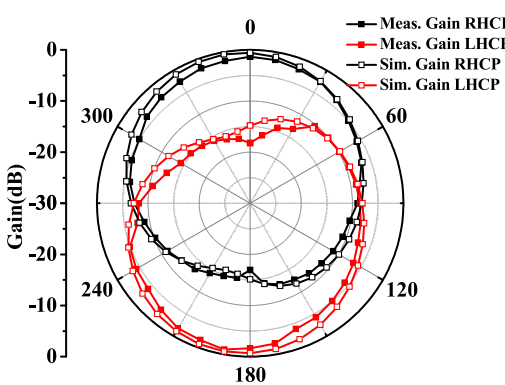

(a)

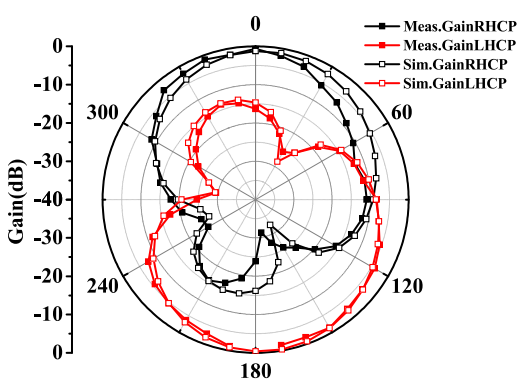

(c)

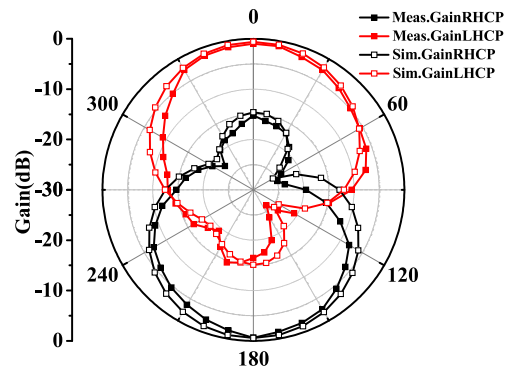

(e)

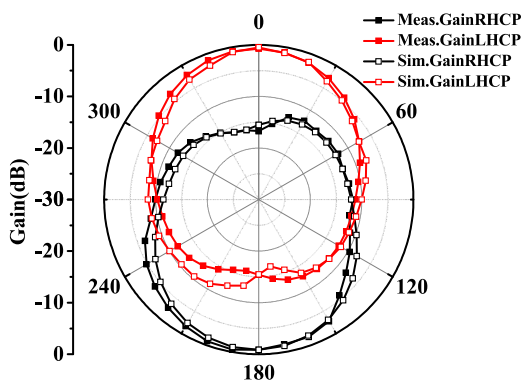

(g)

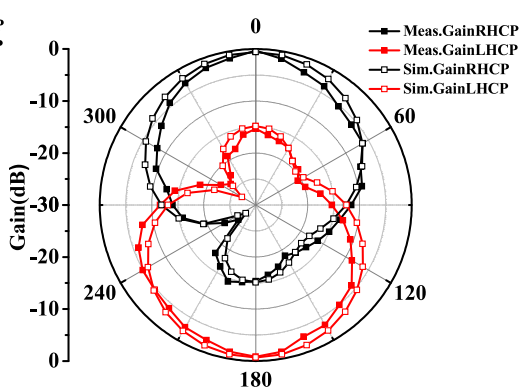

(b)

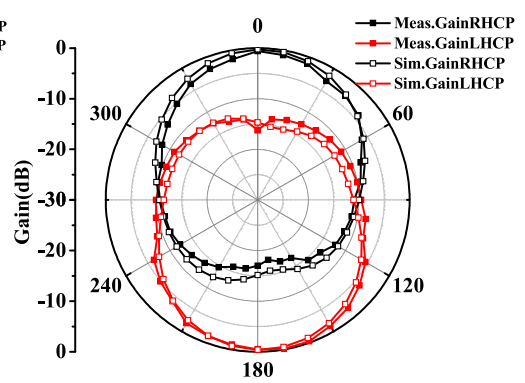

(d)

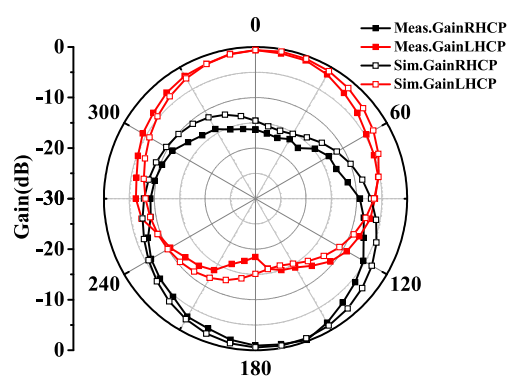

(f)

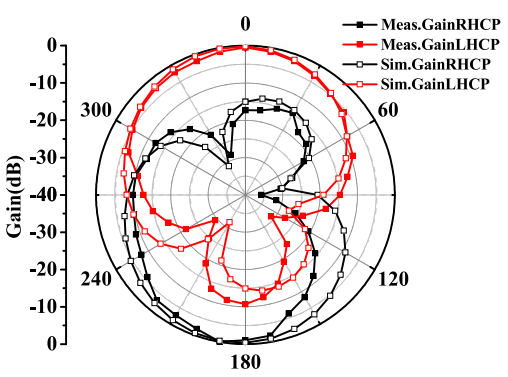

(h)

Fig. 10. Far filed simulated and measured radiation patterns of the proposed antenna: (a) port $1, x-z$ plane at $1.9 \mathrm{GHz}$, (b) port 1 , $y-z$ plane at $1.9 \mathrm{GHz}$, (c) port 2, $x-z$ plane at $1.9 \mathrm{GHz}$, (d) port 2, $y-z$ plane at $1.9 \mathrm{GHz}$, (e) port $1, x-z$ plane at $3.7 \mathrm{GHz}$, (f) port $1, y-z$ plane at $3.7 \mathrm{GHz}$, (g) port $2, x-z$ plane at $3.7 \mathrm{GHz}$ and (h) port $2, y-z$ plane at $3.7 \mathrm{GHz}$. 


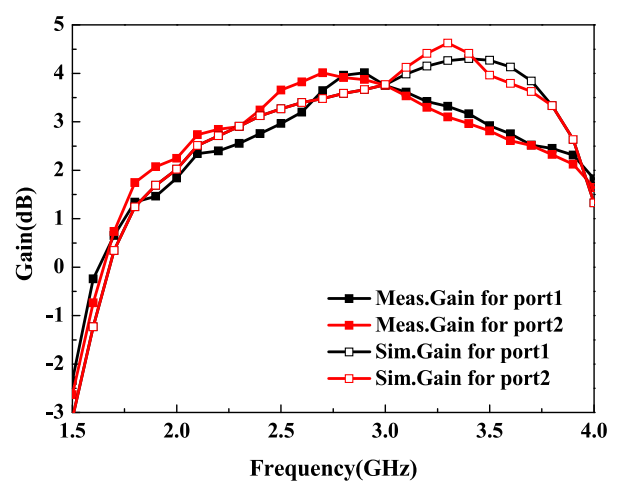

Fig. 11. Simulated and measured results of gain of the proposed antenna.

Table I. Comparison of proposed antenna and references

\begin{tabular}{c|c|c|c|c}
\hline & {$[1]$} & {$[2]$} & {$[8]$} & This work \\
\hline Size $\left(\lambda_{0}{ }^{3}\right)$ & $0.75 * 0.75 * 0.33$ & $0.375 * 0.3 * 0.08$ & $0.56 * 0.56 * 0.015$ & $0.56 * 0.56 * 0.015$ \\
\hline Relative $-10 \mathrm{~dB}$ IMBW & $13 \%$ & $10.8 \%$ & $65.5 \%$ & $76.0 \%$ \\
\hline Relative 3 dB ARBW & $18.7 \%$ & $\begin{array}{c}1.2 \%(\mathrm{LHCP}) \\
1.7 \%(\mathrm{RHCP})\end{array}$ & $59.7 \%$ & $67.0 \%$ \\
\hline Port isolation & $-10 \mathrm{~dB}$ & Not mentioned & $-15 \mathrm{~dB}$ & $-25 \mathrm{~dB}$ \\
\hline
\end{tabular}

\section{Conclusion}

In this letter, a broadband CPW-fed dual CP square slot antenna with modified L-shaped monopoles is proposed, simulated, fabricated and measured. Notching and increasing strips are significant steps in the design procedure for better antenna's performances. Finally, the proposed antenna owns a broad $67 \%$ (from $1.87 \mathrm{GHz}$ to $3.75 \mathrm{GHz}) 3 \mathrm{~dB}$ ARBW, a high port isolation below $-25 \mathrm{~dB}$, a cross polarization level over $-15 \mathrm{~dB}$ and a gain varies from $1.4 \mathrm{~dB}$ to $4.2 \mathrm{~dB}$ within the operating band. Since the proposed antenna is low profile, easy fabricated, dual polarized and owns a broad working band covers Wi-Max $(2.5 \mathrm{GHz}$ to $2.69 \mathrm{GHz}$, $3.2 \mathrm{GHz}$ to $3.8 \mathrm{GHz})$ and WLAN/Bluetooth $(2.4 \mathrm{GHz}$ to $2.484 \mathrm{GHz})$, it can be a suitable and potential candidate for the wireless communication application.

\section{Acknowledgement}

This work was partly supported by the National Defense Pre-Research Foundation of China under grant 3602004, the Open Project Program of the State Key Laboratory of Millimeter Waves under Grant No. K201715, Southeast University, and partly supported by the China Postdoctoral Science Foundation, 2016M600349 and $2017 \mathrm{~T} 100316$. 